\title{
Step-clipping method can improve the detectability of the target lesion in colonic diverticular bleeding $\square$
}

\section{다 (1) $\odot$}

\author{
Authors \\ Taiki Aoyama, Hiroki Takemoto, Yukari Takeuchi, Kenjiro Shigita, Naoki Asayama, Akira Fukumoto, Shinichi Mukai, \\ Shinji Nagata
}

Institution

Department of Gastroenterology, Hiroshima City Asa

Citizens Hospital, Hiroshima, Japan

submitted 2.10.2020

accepted after revision 22.10 .2020

Bibliography

Endoscopy International Open 2021; 09: E356-E362

DOI 10.1055/a-1322-2259

ISSN 2364-3722

(c) 2021. The Author(s).

This is an open access article published by Thieme under the terms of the Creative Commons Attribution-NonDerivative-NonCommercial License, permitting copying and reproduction so long as the original work is given appropriate credit. Contents may not be used for commecial purposes, or adapted, remixed, transformed or built upon. (https://creativecommons.org/licenses/by-nc-nd/4.0/)

Georg Thieme Verlag KG, Rüdigerstraße 14,

70469 Stuttgart, Germany

Corresponding author

Taiki Aoyama, Department of Gastroenterology, Hiroshima City Asa Citizens Hospital, 2-1-1 Kabe-minami, Asakita-ku, Hiroshima 731-0293, Japan

Fax: +81-82-814-1791

t-aoyama@asa-hosp.city.hiroshima.jp

\section{ABSTRACT}

Background and study aims Despite the information regarding extravasation from contrast-enhanced computed tomography (CECT), the detectability of the target diverticulum by colonoscopy remains unsatisfactory in colonic diverticular bleeding (CDB). The lack of common signs recognizable on CT and endoscopic images makes it difficult to set a region of interest; however, this can be overcome with the "step-clipping" method, a strategic marking technique for revealing the target. We aimed to investigate the clinical performance of the step-clipping method in patients with CDB.

Patients and methods Eighty-seven patients diagnosed with CDB with extravasation using CECT who underwent colonoscopy between August 2007 and February 2020 were included. Patients were divided into two categories: the traditional group (those who underwent colonoscopy from August 2007 to May 2017, $n=54$ ) and the step-clipping group (those who underwent colonoscopy from June 2017 to February 2020, $n=33$ ).

Results The detection rate for the responsible diverticulum was significantly higher in the step-clipping group than in the traditional group ( $94 \%$ vs $63 \% ; P=.0013$ ). The step-clipping group had a shorter average search time during colonoscopy ( 9.6 vs 40.5 minutes; $P<.0001$ ) and lower re-bleeding rate after the initial colonoscopy ( $6 \%$ vs $26 \%$; $P=.02)$ than the traditional group, which facilitated earlier hospital discharge (5.4 vs 6.8 days; $P=.027$ ).

Conclusions Colonoscopy using the step-clipping method can improve the detectability of the target lesion within a shorter time, thus leading to lower re-bleeding rates and earlier hospital discharge.

\section{Introduction}

Colonoscopy is strongly recommended as the initial diagnostic procedure for acute lower gastrointestinal bleeding (ALGIB) unless the patient cannot tolerate the bowel preparation and coIonoscopic examination in accordance with the American College of Gastroenterology (ACG) clinical guideline [1]. Moreover, compared with colonoscopy alone, colonoscopy combined with contrast-enhanced computed tomography (CECT), whose diagnostic value for vascular lesions is limited when used alone, pro- vides an additional detectability of $15 \%$ for vascular lesions [25]. In particular, the presence of extravasation from a diverticulum on CECT is definitive evidence of colonic diverticular bleeding (CDB) and provides endoscopists with information regarding the region of interest (ROI) for colonoscopy. However, the specific procedure to use after identification of extravasation on CECT remains controversial. Even with CECT information, the detection rate for the target diverticulum by colonoscopy remains at $50 \%$ to $68.3 \%$ for patients with CDB $[3,4,6]$. This might be because the precise positional information provided 
by CT cannot be obtained with the endoscopic view when spontaneous cessation of bleeding occurs [7]. In other words, the lack of signs commonly recognized on $\mathrm{CT}$ and endoscopic images results in the inability of endoscopists to precisely set the ROI for the colonoscopy examination. Because of individual differences in the shape of the colon, only the cecum and rectum can be located with high confidence in the endoscopic view.

The "step-clipping" method overcomes these limitations by creating artificial signs in the colon using endoclips. These signs can be commonly recognized on CT and endoscopic images, thereby allowing for the formulation of a positional relationship between the clips and the target diverticulum responsible for bleeding [8].

The step-clipping method could be a preferable option in cases of CDB with extravasation on CECT. This study aimed to evaluate whether colonoscopy using the precise positional information provided by the step-clipping method can improve the detectability of the target diverticulum, reduce the endoscopic examination time, and result in favorable clinical outcomes.

\section{Patients and methods}

\section{Patient population}

In total, 693 consecutively hospitalized patients who were diagnosed with ALGIB and presented with hematochezia between August 1, 2007, and February 29, 2020 were included. Among the patients, 422 were diagnosed as having either definitive or presumptive CDB on the basis of colonoscopy findings as the reference standard [5,9]. Definitive CDB was defined as identification of colonic diverticulum with stigmata of recent hemorrhage (SRH), including active bleeding, adherent clot, or nonbleeding visible vessel. Presumptive CDB was defined as colonic diverticula without evidence of SRH but without any other demonstrable cause of bleeding in the colon. All patients with CDB, except those with a contraindication to contrast enhancement agents, were subject to a CECT examination at admission. Although the study population included 34 cases of chronic kidney disease, none of these cases were considered a contraindication for the use of an enhancement agent for the CT examination. This study did not include patients with liver failure or hemodialysis. Three patients were subsequently eliminated because they were considered to have a contraindication to the step-clipping method (presence of extravasation in the cecum and active bleeding during colonoscopy) ( $>$ Fig. 1 ). Therefore, the study population included 87 patients ( 57 men and 30 women; mean age, 75.1 years; range, 34-92 years) who underwent both CECT (with evidence of extravasation) and subsequent colonoscopy at Hiroshima City Asa Citizens Hospital between August 2007 and February 2020. Of these 87 patients, 54 who underwent colonoscopy from August 2007 to May 2017 were classified as the traditional group and 33 who underwent colonoscopy from June 2017 to February 2020 were classified as the step-clipping group.

This study conformed to the principles of the sixth revision of the Declaration of Helsinki (2008), and the study protocol was approved by the Institutional Review Board of Hiroshima

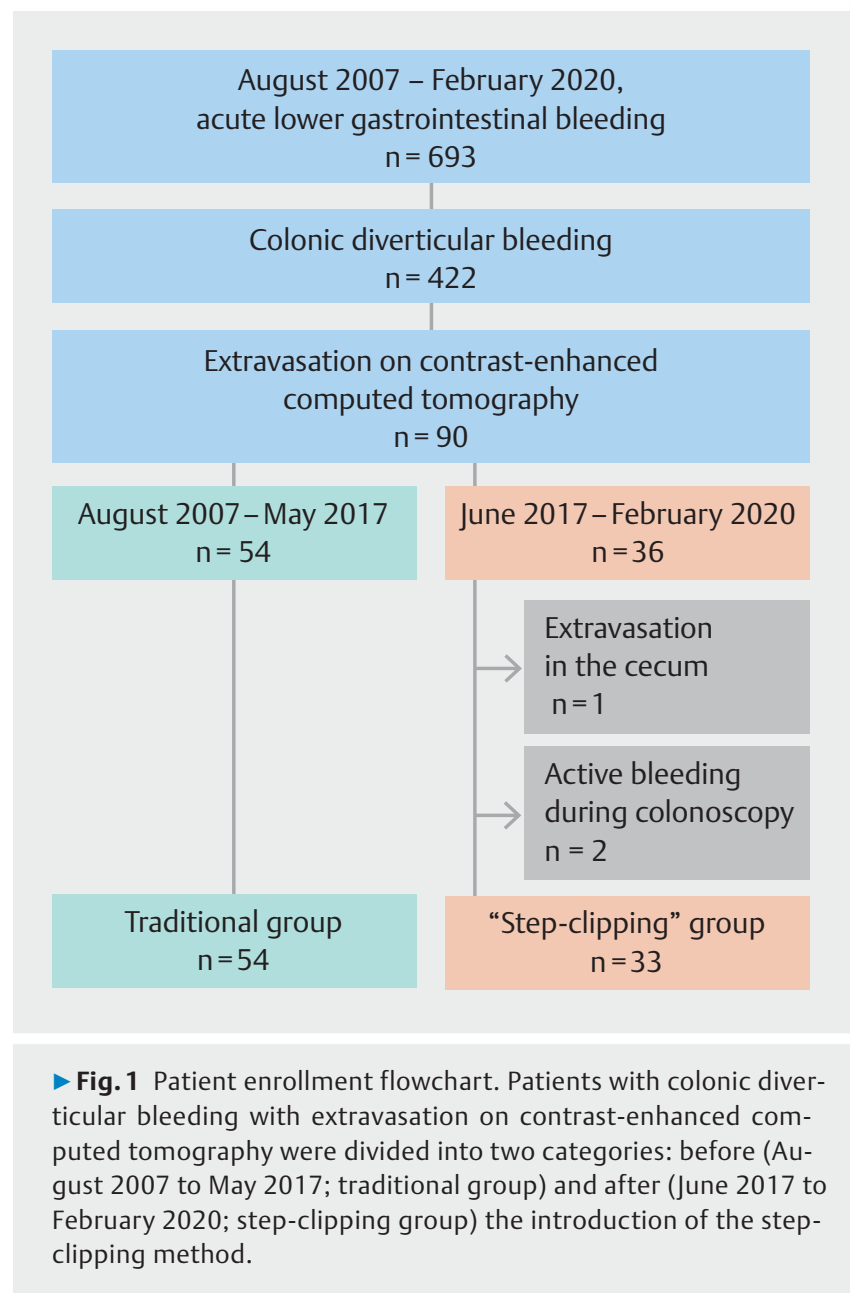

City Asa Citizens Hospital, which also granted permission to access the patients' information on February 26, 2020 (IRB no. 01-6-17). Because of the retrospective design of this study, the requirement for patient consent was waived.

\section{Step-clipping method}

The step-clipping method ( $\triangleright$ Video $\mathbf{1}$ ) is indicated when extravasation is detected on CECT and the site of active bleeding is not identified during the subsequent colonoscopy. After confirming extravasation on CECT, an initial colonoscopy was performed under hemodynamically stable conditions during the day on a weekday regardless of the time of admission, under $\mathrm{CO}_{2}$ insufflation, and after antegrade bowel preparation using $2 \mathrm{~L}$ of polyethylene glycol (PEG). Fluoroscopy was not performed. Minimal search was conducted on initial colonoscopy to prevent changes in the colon environment. An explicit SRH observed during colonoscopy indicates direct hemostasis; thus, the step-clipping method was not necessary. Among those with $\mathrm{SRH}$, direct hemostasis was only indicated if there was active bleeding, in consideration of the presence of residual fake clots in the colon. In cases without evidence of bleeding, the step-clipping method was begun by placing several clips along the longitudinal direction of the colon, 2 inches apart, so that the clip deployment range included the suspi- 


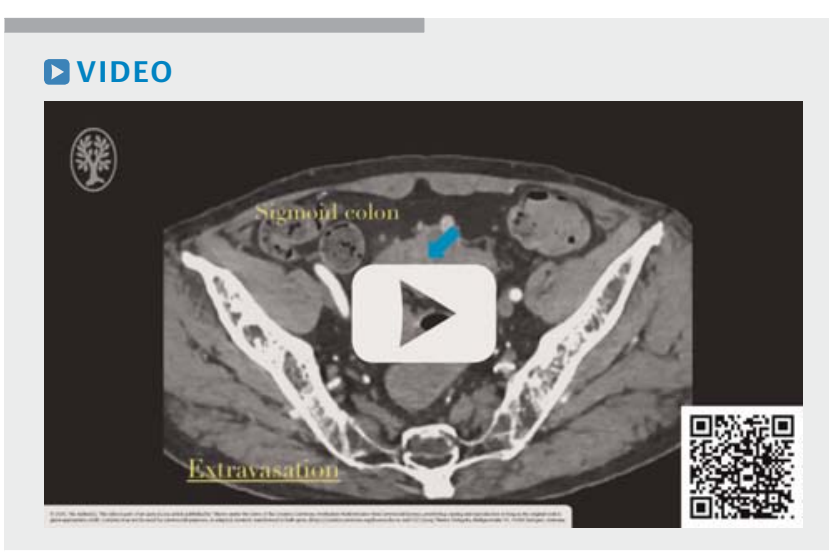

- Video 1 Strategic deployment of clips provides a positional relationship between each clip and the responsible diverticulum, which can be recognized by both computed tomography and endoscopy, and it can guide the examiner to the target lesion during subsequent colonoscopy.

cious site ( $\triangleright$ Fig. $2 \mathbf{2 a}$ ). The range of deployment and number of clips were dependent on the certainty of the target location, which was determined on the basis of the presumed location of the hepatic flexure, splenic flexure, and junction of the sigmoid descending colon in the endoscopic view. Unenhanced CT was conducted 2 hours after placement, which provided sufficient time for complete $\mathrm{CO}_{2}$ absorption or excretion. This step ensured that the colon was deflated, thereby allowing a precise comparison of CT images obtained before and after the procedure. The responsible diverticulum was meticulously tracked and identified on the unenhanced CT image ( $\mathbf{F i g}$. $2 \mathbf{b}$ ) by referring to the initial CECT image obtained at admission ( $\triangleright$ Fig. $2 \mathbf{c}$ ). The target diverticulum was identified on the basis of the consistency of its shape and tracked on the basis of its circumference and distance from a major structure (e.g., the cecum, hepatic flexure, splenic flexure, sigmoid-descending junction) and neighboring symbolic structures (e.g., fecal-impacted diverticula, large diverticula) using 1-mm axial image slices; coronal or sagittal plane images were added as needed. Thereafter, using the unenhanced CT image, the positional relationship between each clip and the target lesion was evaluated. Based on this evaluation, a precise ROI could be set, even in the endoscopic view, during the planning stage ( $\triangleright$ Fig. 3a). A secondary colonoscopy was performed to identify the target diverticulum ( Fig.3b). All colonoscopy examinations and CT image analyses were conducted or closely supervised by expert endoscopists who have performed more than 3000 colonoscopies.

\section{Instruments}

The CT device used a 64-slice diagnostic imaging technique with the Somatom Definition Flash system (Siemens Healthineers, Erlangen, Germany). The colonoscope was a PCFQ260AZI endoscope (Olympus, Tokyo, Japan) with a transparent hood and elastic touch (TOP Corp.) on the tip of the scope. The HX-610-090 endoclip (Olympus) was used.

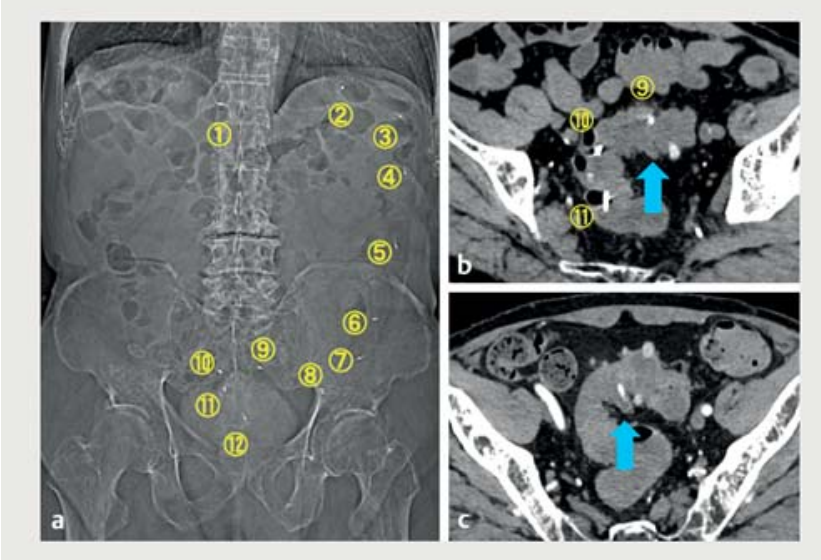

Fig. 2 Computed tomography (CT) images of the step-clipping method a Scout image after clip placement. Twelve clips from the transverse colon to the sigmoid colon are deployed. $\mathbf{b}$ CT axial image after step-clipping. The responsible diverticulum (blue arrow) is observed opposite the ninth clip. c CT axial image before step-clipping, which serves as the reference for locating the responsible diverticulum (blue arrow) with extravasation.

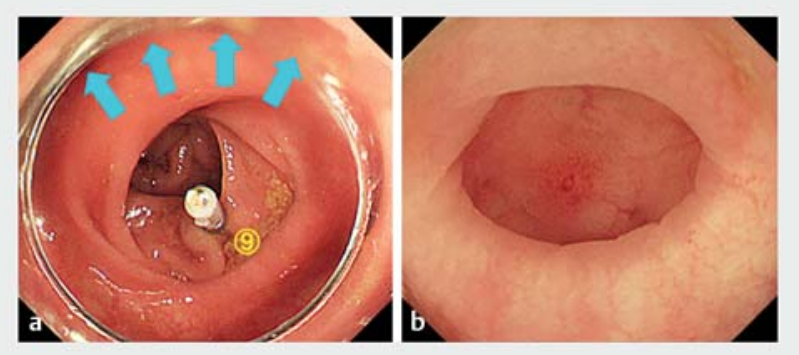

- Fig. 3 Endoscopic management after detailed comparison of computed tomography images. a The responsible diverticulum should be located opposite the ninth clip. The region of interest can be extremely limited (blue arrows) before the second colonoscopy examination. $\mathbf{b}$ The responsible diverticulum is endoscopically identified on the basis of evidence of non-bleeding visible vessel at the dome of the diverticulum.

\section{Study measures}

We compared the demographic and clinical characteristics of the patients in the traditional and step-clipping groups ( $\triangleright \mathrm{Ta}$ ble 1). These included age, sex, body mass index, smoking habits, comorbidities (hypertension, diabetes mellitus, hyperlipidemia, cardiovascular disease, stroke, chronic kidney disease, and chronic obstructive pulmonary disease), medications used (including nonsteroidal anti-inflammatory drugs, antiplatelet agents, and anticoagulant), and bleeding severity, which included a decrease in hematocrit $\geq 20 \%$ at admission and the need for red blood cell (RBC) transfusions during hospitalization. Blood transfusions were performed for patients with a hemoglobin level $\leq 8 \mathrm{~g} / \mathrm{dL}$ or the presence of symptoms indicating inadequate oxygen delivery, consistent with the $A A B B$ guidelines [10]. The features, distribution, and density of the 
- Table 1 Baseline clinical characteristics of the traditional and 'step-clipping' groups.

\begin{tabular}{|c|c|c|c|}
\hline & $\begin{array}{l}\text { Traditional group } \\
n=54\end{array}$ & $\begin{array}{l}\text { Step-clipping group } \\
\mathrm{n}=33\end{array}$ & $P$ value \\
\hline \multicolumn{4}{|l|}{ Demographics } \\
\hline - Age $\geq 75$ years & $32(59)$ & $18(55)$ & .61 \\
\hline - Male sex & $35(65)$ & $22(67)$ & .86 \\
\hline - $B M I \geq 25 \mathrm{~kg} / \mathrm{m}^{2}$ & $10(19)$ & $9(27)$ & .42 \\
\hline - Smoking & $29(54)$ & $16(48)$ & .64 \\
\hline \multicolumn{4}{|l|}{ Comorbidity } \\
\hline - Hypertension & $37(69)$ & $16(48)$ & .06 \\
\hline - Diabetes mellitus & $15(28)$ & $7(21)$ & .49 \\
\hline - Hyperlipidemia & $20(37)$ & $8(24)$ & .22 \\
\hline - Cardiovascular disease ${ }^{1}$ & $26(48)$ & $14(42)$ & .60 \\
\hline - Stroke ${ }^{2}$ & $15(28)$ & $6(18)$ & .31 \\
\hline - Chronic kidney disease ${ }^{3}$ & $24(44)$ & $10(30)$ & .19 \\
\hline - Chronic obstructive pulmonary disease & $3(6)$ & $1(3)$ & .59 \\
\hline \multicolumn{4}{|l|}{ Medication used } \\
\hline - NSAID & $12(22)$ & $8(24)$ & .83 \\
\hline - Antiplatelet agent & $23(43)$ & $11(33)$ & .39 \\
\hline - Anticoagulant & $15(28)$ & $7(21)$ & .49 \\
\hline \multicolumn{4}{|l|}{ Bleeding severity } \\
\hline - Decrease in hematocrit level $\geq 20 \%$ & $7(13)$ & $2(6)$ & .31 \\
\hline - Red blood cell transfusion needed & $28(52)$ & $15(45)$ & .56 \\
\hline \multicolumn{4}{|c|}{$\begin{array}{l}\text { Data are expressed as the number and (\%) of patients. } \\
\text { BMI, body mass index; NSAID, nonsteroidal anti-inflammatory drug. } \\
{ }^{1} \text { Cardiovascular diseases include chronic heart failure, ischemic heart disease, and arteriosclerotic disease. } \\
{ }^{2} \text { Stroke includes cerebral infarction, transient ischemic attack, and cerebral hemorrhage. } \\
{ }^{3} \text { Sustained renal malfunction was defined as an estimated glomerular filtration rate }<60 \mathrm{~mL} / \mathrm{min} / 1.73 \mathrm{~m}^{2} \text {. }\end{array}$} \\
\hline
\end{tabular}

diverticula and the location of the responsible diverticulum of the study population were compared between the groups ( Table 2).

The endoscopic management and clinical course of the two groups were also compared ( $\downarrow$ Table 3 ). The major outcomes of the study were the endoscopic detection rate and total search time for the responsible diverticulum. The total examination time during colonoscopy was also assessed, which comprised duration of scope insertion, time searching for the target lesion, and treatment during the endoscopy examination. The clinical course consisted of the re-bleeding rate, RBD transfusion rate after the initial colonoscopy, and duration between the initial colonoscopy and discharge. Re-bleeding was defined as significant fresh blood excretion after initial colonoscopy with any of the following: hemorrhagic shock, the need for transfusion, or identification of blood pooling on further colonoscopy.

\section{Statistical analysis}

All statistical analyses were performed using JMP version 9 (SAS Institute Inc., Cary, North Carolina, United States). An unpaired $t$-test was used to compare the quantitative data between the groups, and a chi-square test was used to compare the categorical data. Yate's correction and Fisher's exact test were used when required. A two-sided $P<.05$ was considered statistically significant.

\section{Results}

There were no statistically significant differences in demographics, comorbidities, medications used, or bleeding severity between the two groups. Fifteen patients with hemodynamic instability at admission were administered intravenous fluid resuscitation or RBC transfusion and achieved hemodynamic stability. More cases of left-side colonic diverticula were observed in the step-clipping group than in the traditional group; how- 
- Table 2 Baseline characteristics of the colonic diverticula of the traditional and "step-clipping" groups.

\begin{tabular}{|c|c|c|c|}
\hline & $\begin{array}{l}\text { Traditional group } \\
\mathrm{n}=54\end{array}$ & $\begin{array}{l}\text { Step-clipping group } \\
\mathrm{n}=33\end{array}$ & $P$ value \\
\hline \multicolumn{4}{|l|}{ Distribution of diverticula } \\
\hline - Right side of the colon & $40(74)$ & $27(82)$ & .40 \\
\hline - Left side of the colon & $35(65)$ & $29(88)$ & .02 \\
\hline \multicolumn{4}{|l|}{ Location of extravasation on CECT } \\
\hline - Ascending colon & $30(56)$ & $16(49)$ & \multirow[t]{4}{*}{.39} \\
\hline - Transverse colon & $6(11)$ & $3(9)$ & \\
\hline - Descending colon & $5(9)$ & $4(12)$ & \\
\hline - Sigmoid colon & $13(24)$ & $10(30)$ & \\
\hline Density of diverticula (per 2 inches) $\geq 10$ & $24(44)$ & $17(52)$ & .52 \\
\hline \multicolumn{4}{|l|}{$\begin{array}{l}\text { Data are expressed as the number and (\%) of patients. } \\
\text { CECT, contrast-enhanced computed tomography. }\end{array}$} \\
\hline & $\begin{array}{l}\text { Traditional group } \\
\mathrm{n}=54\end{array}$ & $\begin{array}{l}\text { Step-clipping group } \\
\mathrm{n}=33\end{array}$ & $P$ value \\
\hline $\begin{array}{l}\text { Duration between initial colonoscopy and admission, hours } \\
\text { Mean [SD] }\end{array}$ & $16.9[18.4]$ & $28.3[20.7]$ & .0045 \\
\hline Detection of the responsible diverticulum, n (\%) & $34(63)$ & $31(94)$ & .0013 \\
\hline \multicolumn{4}{|l|}{ Total examination time during colonoscopy, minutes } \\
\hline - Mean [SD] & $64.7[40.3]$ & $38.4[17.9]$ & \multirow[t]{2}{*}{.0006} \\
\hline - Median (range) & $55(23-250)$ & $35(16-85)$ & \\
\hline \multicolumn{4}{|l|}{ Total search time during colonoscopy, minutes } \\
\hline - Mean [SD] & $40.5[38.9]$ & $9.6[14.5]$ & \multirow[t]{2}{*}{$<.0001$} \\
\hline - Median (range) & $30(3-235)$ & $4(1-58)$ & \\
\hline \multicolumn{4}{|l|}{ Treatment, n (\%) } \\
\hline - Endoscopic band ligation & $27(50)$ & $29(85)$ & .0003 \\
\hline - Clipping & $7(13)$ & $2(6)$ & .31 \\
\hline - Conservative treatment & $20(37)$ & $2(6)$ & .0013 \\
\hline \multicolumn{4}{|l|}{ Clinical outcome } \\
\hline - Re-bleeding after initial colonoscopy, n (\%) & $14(26)$ & $2(6)$ & .020 \\
\hline - Blood transfusion needed after initial colonoscopy, n (\%) & $14(26)$ & $2(6)$ & .020 \\
\hline $\begin{array}{l}\text { - Duration between initial colonoscopy and discharge, days } \\
\text { Mean [SD] }\end{array}$ & $6.8[2.9]$ & $5.4[2.2]$ & .027 \\
\hline
\end{tabular}

ever, there was no difference in the location of the responsible lesion between the groups.

The traditional group comprised patients who did not undergo the step-clipping method. Therefore, the timing of the initial colonoscopy and bowel preparation (PEG administration or glycerin enema) were dependent on the decision of the chief physician. In the traditional group, 17 patients underwent colo- noscopy within 24 hours of admission under bowel preparation with glycerin enema; SRH was successfully identified and endoscopically treated in nine patients, and the other eight patients in whom SRH was not identified underwent further colonoscopy at the earliest timing with PEG preparation. Bowel preparation was conducted until the rectal effluent became clear of stool to ensure unobstructed observation of the colonic diverti- 
cula. The average duration between the initial colonoscopy and admission was shorter in the traditional group than that in the step-clipping group (16.9 vs 28.3 hours; $P=.0045$ ).

The rate of detection of the responsible diverticulum for the step-clipping group was significantly higher than that for the traditional group ( $94 \%$ vs $63 \% ; P=.0013$ ). Furthermore, the average search time during colonoscopy was shorter for the step-clipping group than that for the traditional group (9.6 vs 40.5 minutes; $P<.0001)$. The median number of deployed clips was eight (range, 3-12) in the step-clipping group. SRH was identified during colonoscopy in 34 patients in the traditional group: active bleeding in 22 patients (41\%), adherent clots in 8 patients (15\%), and non-bleeding visible vessels in 4 patients (7\%). SRH was identified in 31 patients in the step-clipping group: adherent clots in 11 patients (33\%) and non-bleeding visible vessels in 20 patients (61\%). All patients with SRH identified during colonoscopy initially underwent endoscopic treatment. Clipping was the first choice before November 2000, and endoscopic band ligation (EBL) was the first choice after November 2011; however, when EBL failed, clipping was attempted. Although more cases required endoscopic treatment in the step-clipping group, the average total examination time during colonoscopy in the step-clipping group was shorter than that in the traditional group (38.4 vs 64.7 minutes; $P=.0006$ ). A favorable clinical course was achieved by the step-clipping group, with its lower re-bleeding rate and lesser need for RBC transfusions after the initial colonoscopy than the traditional group ( $6 \%$ vs $26 \% ; P=.02$ ), which facilitated earlier discharge (5.4 vs 6.8 days; $P=.027)$. Two patients in the step-clipping group experienced recurrent brisk bleeding after the initial colonoscopy. The one case was refractory to endoscopic treatment (EBL and clipping) and proceeded to transcatheter arterial embolization (TAE). The other case was attributable to failure during repeat colonoscopies and subsequent angiography, thus requiring surgical resection. Both cases required RBC transfusion for hemodynamic resuscitation. No adverse events related to the endoscopic examination occurred.

\section{Discussion}

There is a high rate of spontaneous hemostasis with CDB (70\%$90 \%$ ) [11]. However, $20 \%$ to $30 \%$ and $30 \%$ to $40 \%$ of patients with CDB experienced re-bleeding at 1 year and at 2 years, respectively, when only conservative treatment was provided [12-14]. Identification of the responsible lesion by colonoscopy was a preventive factor for re-bleeding and reportedly reduced approximately $80 \%$ of re-bleeding cases during a median observation period of 17.5 months [15]. Moreover, a lower re-bleeding rate may be attributed to the emergence of promising endoscopic treatment, particularly ligation therapy. Ligation therapy contributes to the high primary success rate for hemostasis $(100 \%)$ and the low re-bleeding rate with early timing (9\%) [16-18]. However, it is indicated only when the target diverticulum is identified. Higher detectability rates and steady endoscopic treatment could result in a favorable clinical course. The step-clipping group had an extremely high rate of target detection, and most targets were subjected to the liga- tion method, which resulted in significantly lower re-bleeding rates and fewer blood transfusions, thereby facilitating earlier discharge than that in the traditional group.

Identification of extravasation on CECT provides $24 \%$ to $48 \%$ additional detectability of the target diverticulum during the colonoscopic examination compared with cases without observable extravasation $[3,4,6]$; however, extravasation-positive rates are not high for patients with CDB (range, $25 \%$ to $36 \%$ ) [3-5]. Compared with the diagnosis rates of the target lesion of $92 \%$ to $98 \%$ for acute nonvariceal upper gastrointestinal bleeding [19], the detectability rates of $50 \%$ to $68.3 \%$ for CBD, even with the help of extravasation identification, remain unsatisfactory. We successfully improved the detectability of SRH from $63 \%$ to $94 \%$ using the step-clipping method in patients with CBD with extravasation identified on CECT.

The ACG guideline strongly recommends performing urgent colonoscopy within 24 hours of admission to achieve a higher rate of SRH detection in cases of ALGIB [1]. Because of the multiple procedures involved in the step-clipping method and the fact that its conduct was limited to weekdays, the timing of initial colonoscopy resulted in delay with the step-clipping method compared with the traditional method. Nonetheless, higher identification rates for SRH were achieved using the step-clipping method than with the traditional method.

Because of the low detection rate for the target diverticulum using endoscopy, the examination can be extended to 45 to 140 minutes, thus leading to troublesome examinations [2022]. The detectability of the target diverticulum during colonoscopy depends on the skill of the endoscopist and an element of chance. However, the step-clipping method serves as a navigation map in the colon [23], thus lessening reliance on the skill of the endoscopist. Because the step-clipping technique is based on analysis of CT images, the method allows endoscopists the time needed to examine the colon without the stress associated with prolonged endoscopic examination. CT-based preplanning eliminates redundant searching for the target lesion during the second colonoscopy. In our study, the step-clipping group required a search time of only 1 to $58 \mathrm{~min}$ utes (median, 4 minutes), resulting in a detectability rate of $94 \%$, whereas the traditional group required a search time of 3 to 235 minutes (median, 30 minutes), resulting in a detectability rate of $63 \%$. The step-clipping method requires insertion of the colonoscope twice, which is troublesome; however, the total examination time for colonoscopy is significantly shorter than that using the traditional method (median, 9.6 vs 40.5 minutes).

In the step-clipping group, most SRH cases found by colonoscopy were non-bleeding visible vessels. Because $55 \%$ of the population is subjected to elective colonoscopy, it is rare to encounter active bleeding. A non-bleeding vessel is tiny and difficult to detect; however, a limited ROI and endoscopic management such as image enhancement endoscopy or underwater observation could improve the detectability of such a lesion [24-26].

We acknowledge that this study has some limitations. First, the step-clipping method requires additional costs (approximately $€ 230$ ) and radiation exposure (approximately $5 \mathrm{mGy}$ ) 
for secondary colonoscopy and unenhanced CT compared with the traditional approach combining CECT and colonoscopy. Although this may seem to be inconvenient, consistent detection and treatment of target lesions might reduce rates of re-bleeding and readmission, thus lowering costs and radiation exposure in the long term. Second, the step-clipping method can only be applied when extravasation is identified on CECT. Therefore, the clinical impact on reduction in mortality from the disease, which has been reported to be up to $3.9 \%$ [27, 28], remains obscure. Moreover, extensive discussion about the performance of subsequent endoscopic treatment is needed to determine whether the step-clipping method reduces the need for TAE and surgery. Third, the step-clipping method includes multiple procedures. Not all patients with CDB who have extravasation on CECT may be candidates for the method. It would be expedient to apply the step-clipping strategy to patients when the initial colonoscopy missed the responsible lesion after extensive search. Fourth, this study involved a limited population at a single center. Multiple large-scale cohorts and long-term studies are necessary to verify the clinical impact of the step-clipping method.

\section{Conclusion}

Colonoscopy involving precise positional information using the step-clipping method can improve the detectability of target lesions within a short time, thus leading to lower re-bleeding rates and facilitating earlier discharge.

\section{Competing interests}

The authors declare that they have no conflict of interest.

References

[1] Strate LL, Gralnek IM. ACG clinical guideline: management of patients with acute lower gastrointestinal bleeding. Am J Gastroenterol 2016; 111: 459-474

[2] Nagata N, Niikura R, Aoki T et al. Role of urgent contrast-enhanced multidetector computed tomography for acute lower gastrointestinal bleeding in patients undergoing early colonoscopy. J Gastroenterol 2015; $12: 1162-1172$

[3] Nakatsu S, Yasuda H, Maehata T et al. Urgent computed tomography for determining the optimal timing of colonoscopy in patients with acute lower gastrointestinal bleeding. Intern Med 2015; 54: 553-558

[4] Sugiyama T, Hirata Y, Kojima Y et al. Efficacy of contrast-enhanced computed tomography for the treatment strategy of colonic diverticular bleeding. Intern Med 2015; 54: 2961-2967

[5] Umezawa S, Nagata N, Arimoto J et al. Contrast-enhanced CT for colonic diverticular bleeding before colonoscopy: a prospective multicenter study. Radiology 2018; 288: 755-761

[6] Obana T, Fujita N, Sugita R et al. Prospective evaluation of contrastenhanced computed tomography for the detection of colonic diverticular bleeding. Dig Dis Sci 2013; 58: 1985-1490

[7] Niikura R, Nagata N, Shimbo T et al. Colonoscopy can miss diverticula of the left colon identified by barium enema. World J Gastroenterol 2013; 19: 2362-2367
[8] Tamari H, Aoyama T, Nagata S et al. "Step-clipping" method: a technique for detecting previously bleeding diverticula. Endoscopy 2018; 50: E10-E12

[9] Jensen DM, Machicado GA, Jutabha R et al. Urgent colonoscopy for the diagnosis and treatment of sever diverticular hemorrhage. N Engl J Med 2000; 342: 78-82

[10] Carson JL, Grossman B], Kleinman S et al. Red blood cell transfusion: a clinical practice guideline from the AABB. Ann Intern Med 2012; 157 : 49-58

[11] Kaise M, Nagata N, Ishii $\mathrm{N}$ et al. Epidemiology of colonic diverticula and recent advances in the management of colonic diverticular bleeding. Dig Endosc 2020; 32: 240-250

[12] Aytac E, Stocchi L, Gorgun E et al. Risk of recurrence and long-term outcomes after colonic diverticular bleeding. Int J Colorectal Dis 2014; 29: 373-378

[13] Okamoto T, Watabe H, Yamada A et al. The association between arteriosclerosis related diseases and diverticular bleeding. Int J Colorectal Dis 2012; 27: 1161-1166

[14] Nishikawa H, Maruo T, Tsumura T et al. Risk factors associated with recurrent hemorrhage after the initial improvement of colonic diverticular bleeding. Acta Gastroenterol Belg 2013; 76: 20-24

[15] Wada M, Kato M, Hirai Y et al. Initial management of colonic diverticular bleeding: observational study. Digestion 2018; 98: 41-47

[16] Nagata N, Ishii N, Kaise M et al. Long-term recurrent bleeding risk after endoscopic therapy for definitive colonic diverticular bleeding: band ligation versus clipping. Gastrointest Endosc 2018; 88: 841-853

[17] Kobayashi K, Furumoto Y, Akutsu D et al. Endoscopic detachable snare ligation improves the treatment for colonic diverticular hemorrhage. Digestion 2019; 6: 1-9

[18] Akutsu D, Narasaka T, Wakayama M et al. Endoscopic detachable snare ligation: a new treatment method for colonic diverticular hemorrhage. Endoscopy 2015; 47: 1039-1042

[19] Jaskolka JD, Binkhamis S, Prabhudesai V et al. Acute gastrointestinal hemorrhage: radiologic diagnosis and management. Can Assoc Radiol J 2013; 64: 90-100

[20] Shimamura Y, Ishii N, Omata F et al. Endoscopic band ligation for colonic diverticular bleeding: possibility of standardization. Endosc Int Open 2016; 4: E233-E247

[21] Yen EF, Ladabaum U, Muthusamy VR et al. Colonoscopic treatment of acute diverticular hemorrhage using endoclips. Dig Dis Sci 2008; 53 : 2480-2485

[22] Farrell J], Graeme-Cook F, Kelsey PB. Treatment of bleeding colonic diverticula by endoscopic band ligation: an in-vivo and ex-vivo pilot study. Endoscopy 2003; 35: 823-829

[23] Tamari H, Aoyama T, Shigita K et al. The 'step-clipping' method: a guide for identifying a previously bleeding colonic diverticulum. Endosc Int Open 2020; 8: E64-E69

[24] Aoyama T, Tamari H, Nagata S. Underwater narrow-band imaging in colonic diverticular bleeding. Intern Med 2020; 59: 137-138

[25] Kishimoto T, Kitaichi T, Knemasa K. Usefulness of water immersion observation to identify the stigmata of hemorrhage in colonic diverticular bleeding. Dig Endosc 2018; 30: 121-122

[26] Tamari H, Aoyama T, Fukumoto A et al. Application of linked color imaging for detecting a previously bleeding colonic diverticulum. Video GIE 2018; 3: 319-321

[27] Niikura R, Yasunaga H, Yamaji Y et al. Factors affecting in-hospital mortality in patients with lower gastrointestinal tract bleeding: a retrospective study using a national database in Japan. J Gastroenterol 2015; 50: 533-540

[28] Strate LL, Ayanian JZ, Kotler G et al. Risk factors for mortality in lower intestinal bleeding. Clin Gastroenterol Hepatol 2008; 6: 1004-1010 